\title{
Comparison of Ultrasonography and Computed Tomography to Evaluate the Causes of Biliary Obstruction
}

\author{
Naffisa Adedin, Abdullah Shahriar, Akhtar Uddin Ahmed, AS Mohiuddin, Jafreen Sultana \\ Nusrat Ghafoor, Nayeema Rahman
}

\begin{abstract}
Objective: To observe the role of computed tomographic (CT) scan and ultrasonography (USG) examination to evaluate the causes of biliary obstruction.
\end{abstract}

Materials and methods: This cross-sectional study was conducted in a total of 57 patients clinically suspected of obstructive jaundice.

Results: The highest incidence of biliary obstruction was found in the age group between 40 and 49 years and the mean $( \pm S E)$ age of the patients was $48.4 \pm 1.6$ years. Serum bilirubin and serum alkaline phosphatase were high in all patients. For evaluation of pancreatic mass, USG found true positive in 13 with no false positive, false negative 2 and true negative 42 cases. Similarly, CT scan found true positive in 15 and no false positive, no false negative and true negative in 42 cases. Sensitivity and specificity of USG in detecting pancreatic mass were 80 and $97.6 \%$. CT scan showed $93.3 \%$ sensitivity and $97.6 \%$ specificity. In case of gallbladder (GB) mass USG found true positive in 20 and 1 false positive. There was no false negative and true negative in 36 cases. CT scan also revealed better sensitivity. USG could not detect any case of periampulla.

Conclusion: Accuracy of USG and CT is high in detecting biliary tree dilatation, with CT scan slightly more accurate than USG. The difference in cost between the two is likely to decline with time and make CT even more attractive and handy for imaging the hepatobiliary system.

Keywords: Biliary obstruction, Ultrasonography, Computed tomography.

How to cite this article: Adedin N, Shahriar A, Ahmed AU, Mohiuddin AS, Sultana J, Ghafoor N, Rahman N. Comparison of Ultrasonography and Computed Tomography to Evaluate the Causes of Biliary Obstruction. Euroasian J Hepato-Gastroenterol 2012;2(2):98-103.

Source of support: Nil

Conflict of interest: None

\section{INTRODUCTION}

The role of imaging in patients with suspected bile duct obstruction is not only to confirm the presence of biliary obstruction, but also to determine the level and cause of obstruction and the extent or stage of the disease process. ${ }^{1,2}$ Detailed information for diagnosis of biliary obstruction is usually obtained by the combined use of ultrasonography (USG), computed tomography (CT), endoscopic retrograde cholangiopancreatography (ERCP) and percutaneous transhepatic cholangiography (PTC). There have been added magnetic resonance cholangiopancreatography (MRCP) and cholangio computed tomography (CCT).
USG is $95 \%$ accurate to detect dilated and nondilated bile ducts, if the serum bilirubin exceeds $170 \mu \mathrm{mol} / \mathrm{l}$ $(10 \mathrm{mg} / \mathrm{dl}) .{ }^{3}$ False negatives are seen if the obstruction is of short duration or intermittent. Diagnostic procedures using ultrasound are painless, harmless, relatively inexpensive, available and there is no ionizing radiation. But it has some limitations in the diagnosis of some of the causes of biliary obstruction. The larger main right and left hepatic ducts can be identified in USG as tubular structures running anterior and parallel to the right and left branches of the portal vein, and measures up to $2 \mathrm{~mm}$ in diameter in the nondilated system. ${ }^{4}$ The diameter of the normal common duct at the porta hepatis should be less than $5 \mathrm{~mm},{ }^{5}$ increasing slightly (less than $6 \mathrm{~mm}$ ) as the duct runs caudally in the free edge of the lesser omentum and within the head of the pancreas. ${ }^{6}$

$\mathrm{CT}$ is another imaging modality to evaluate obstructive jaundice. The overall accuracy of CT for diagnosing biliary obstruction has been reported at 85 to $97 \%{ }^{7}$ sensitivity $96 \%$ and specificity $91 \%{ }^{8}$ Diagnosis of extrahepatic bile duct dilatation is based on the caliber of the common hepatic duct (CHD) and common bile duct (CBD). On CT, a CBD with diameter less than or equal to $7 \mathrm{~mm}$ should be considered normal, between 7 and $10 \mathrm{~mm}$ equivocal and greater than $10 \mathrm{~mm}$ dilated. ${ }^{8}$ Determination of the etiology of the obstruction requires detailed evaluation of the appearance of the transition zone.

In Bangladesh, biliary obstruction is one of the common clinical entity. USG and CT scan are two important diagnostic tools available in our country to evaluate biliary tree. Study was designed to demonstrate the role of CT and USG in the evaluation of biliary obstruction and its cause in the prospective of our country.

\section{MATERIALS AND METHODS}

This study was carried out in the Department of Radiology and Imaging of Bangladesh Institute of Research and Rehabilitation of Endocrine and Metabolic Disorder (BIRDEM) in collaboration with the Department of Hepatobiliary Surgery and Histopathology Department of the same institute from January 2005 to December 2006. This crosssectional study included 72 clinically suspected hospital admitted patients of obstructive jaundice aged from 23 to 72 years. Fifteen patients were excluded due to unfit/refused 
surgery or ERCP or as USG and CT scan both not done, histopathological/ERCP report not collected and drop out cases. During reviewing their clinical history special emphasis was given on upper abdominal pain, itching, jaundice, upper abdominal mass and weight loss. Physical examination was done regarding jaundice, any upper abdominal mass, tenderness, condition of surrounding structures and lymph node involvement by concerned physician. Then they were routinely investigated using appropriate hematological and biochemical parameters to come to the clinical diagnosis. Finally, 57 patients were included in the study. Prior to the commencement of this study, the research protocol was approved by the ethical review committee of BIRDEM. The objectives of the study along with its procedure, risks and benefits of this study were explained to the patients and then informed consent was taken. Data were collected in predesigned structured data collection sheets (proforma).

\section{Positioning of the Patients and Probe Orientation}

An initial survey of the biliary tree and gallbladder with the patient in supine position and also in left lateral decubitus was done.

During scanning, the size of intrahepatic biliary tree, extrahepatic biliary tree, main pancreatic duct and gallbladder, lumen of the gallbladder and common bile duct, pancreas, all were searched for presence of any mass lesion, calculus or any other pathology. Periampullary region was tried to be assessed. Lymphadenopathy, ascites, ascaris, etc were also tried to find out. The postoperative resected tissues were examined histopathologically in the respective histopathology department and then collected reports were correlated with USG and CT scan findings. In respective cases ERCP reports were collected and also correlated with USG and CT scan findings.

\section{STATISTICAL ANALYSIS}

Statistical analysis was done by computer software devised as the statistical package for social science (SPSS ver 16.0). The results were presented in tables and diagrams. The sensitivity, accuracy, positive predictive values and negative predictive values of ultrasonogram and CT in diagnosis of cause of biliary obstruction were calculated out. A p-value $<0.05$ was considered as significant.

\section{RESULTS}

The highest incidence of biliary obstruction was found in the age group between 40 and 49 years and the mean $( \pm$ SE) age of the patients was $48.4 \pm 1.6$ years with range from 23 to 72 years. Female patients were 28 (49.1\%) and male were 29 (50.9\%). The representative feature of CT and USG has been shown in Figures 1 and 2, respectively. The levels of serum bilirubin (1.8-27 mg/dl) and serum alkaline phosphatase (168-1,795 U/l) were high in all patients. The relative ratios of patients according to clinical features have been cited in Figure 3.

As shown in Table 1, USG and CT could not detect cause of biliary obstruction in 18 (31.6\%) and 2 (3.5\%) cases respectively.

The utility of CT and USG to diagnose biliary obstruction have been shown in Table 2 .

Overall, USG could detect cause of biliary obstruction in $39(68.4 \%)$ cases and could not detect cause of biliary obstruction in 18 (31.6\%) cases. CT scan could detect causes of biliary obstruction in 55 (96.5\%) cases. Only 2 (3.5\%) cases were not detected at CT scan. The difference in results was statistically significant $(\mathrm{p}<0.001)$.

In USG for identification of cause of biliary obstruction out of the 57 cases, true positive 39 and no false positive,

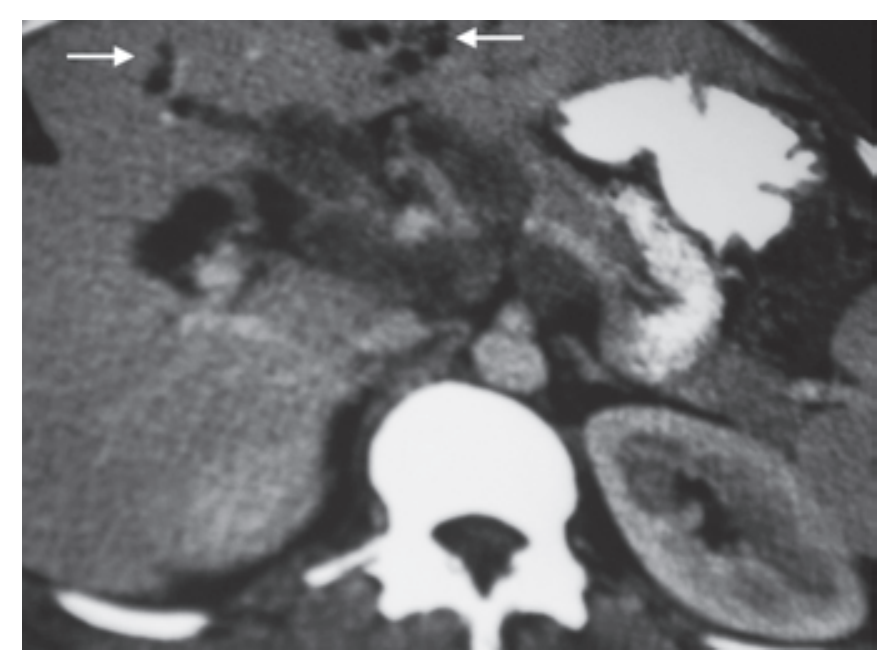

Fig. 1: CT scan showing dilated intrahepatic tree

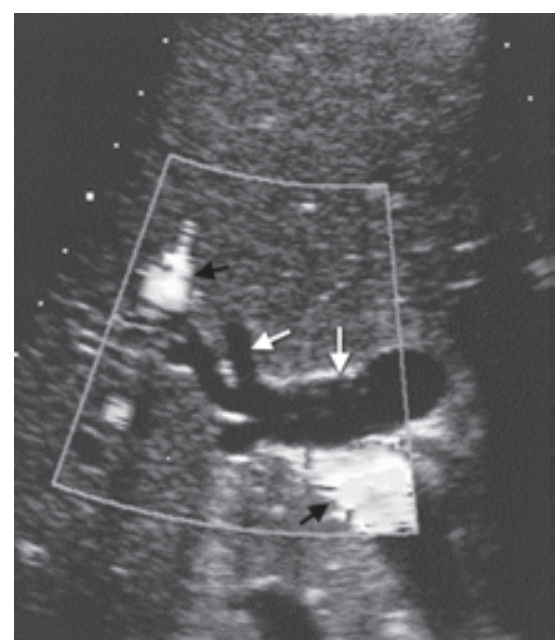

Fig. 2: USG shows dilated bile ducts (white arrows) which are seen as tortuous tubular structures in the liver. Color Doppler makes differentiation of bile ducts (white arrows) and blood vessels (black arrows) 


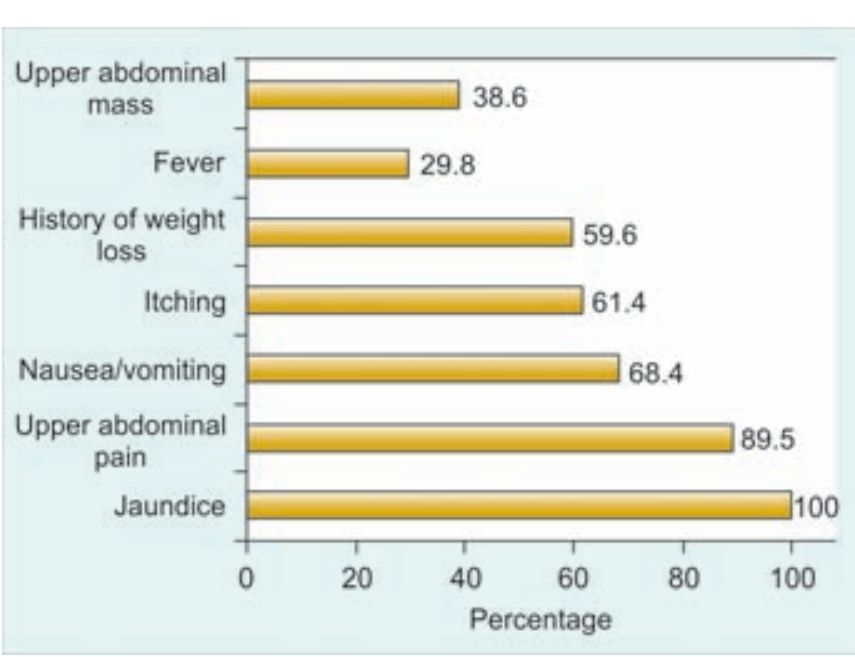

Fig. 3: Distribution of patients according to clinical features

18 false negative and no true negative cases were there. Similarly, CT scan found true positive 55 and no false positive, 2 false negative and no true negative case (Table 3). Sensitivities of USG and CT scan in this aspect were 68.4 and $96.5 \%$ respectively. Accuracy of these investigations were also 68.4 and $96.5 \%$ respectively. PPV of both of these examinations were $100 \%$, whereas NPV of USG and CT scan were also similar, which was $0 \%$.

The present study findings indicate that both USG and CT scan are ideal, noninvasive, safe imaging modalities for diagnosis of biliary dilatation. However, CT scan is far superior in detecting the cause of biliary obstruction.

\section{DISCUSSION}

The imaging appearance of different causes of obstructive jaundice in USG and CT scan and comparison of the accuracy of USG and CT scan in diagnosing the causes of biliary obstruction have not yet been evaluated in our country.

In previous study, the mean age of presentation of biliary obstruction was $48.14 \pm 12.55$ years and ranged from 15 to 72 years. Most of the patients were in the 5th decade of life, which closely agree with the present study. ${ }^{9}$ The maximum number that is 25 (43.9\%) cases were found in the age group of 40 to 49 years.

As regarding sex incidence of biliray obstruction, no significant difference was found in the occurrence of

Table 1: Distribution of patients according to ultrasonographic, computed tomographic and ultimate diagnosis $(n=57)$

\begin{tabular}{|c|c|c|c|c|c|c|}
\hline & \multicolumn{2}{|c|}{ USG } & \multicolumn{2}{|c|}{$C T$} & \multicolumn{2}{|c|}{ Ultimate diagnosis } \\
\hline & $n$ & $\%$ & $n$ & $\%$ & $n$ & $\%$ \\
\hline No diagnosis & 18 & 31.6 & 2 & 3.5 & 0 & 0.0 \\
\hline Periampullary carcinoma & 0 & 0.0 & 10 & 17.5 & 10 & 17.5 \\
\hline Cholangiocarcinoma & 0 & 0.0 & 2 & 3.5 & 2 & 3.5 \\
\hline $\mathrm{Ca} / \mathrm{mass}$ in pancreas & 13 & 22.8 & 15 & 26.3 & 15 & 26.3 \\
\hline GB mass & 21 & 36.8 & 20 & 35.1 & 20 & 35.1 \\
\hline Choledocholithiasis & 5 & 8.8 & 6 & 10.5 & 8 & 14.0 \\
\hline Benign stricture & 0 & 0.0 & 1 & 1.8 & 1 & 1.8 \\
\hline Choledochal cyst & 0 & 0.0 & 1 & 1.8 & 1 & 1.8 \\
\hline Total & 57 & 100 & 57 & 100 & 57 & 100 \\
\hline
\end{tabular}

Table 2: Distribution of patients according to the ability of USG and CT scan to diagnose cause of biliary obstruction ( $\mathrm{n}=57$ )

\begin{tabular}{|c|c|c|c|c|c|}
\hline & \multicolumn{2}{|c|}{ USG } & \multicolumn{2}{|c|}{$C T$} & \multirow[t]{2}{*}{$p$-value } \\
\hline & $n$ & $\%$ & $n$ & $\%$ & \\
\hline No diagnosis & 18 & 31.6 & 2 & 3.5 & $0.001 \mathrm{~S}$ \\
\hline Diagnosed & 39 & 68.4 & 55 & 96.5 & \\
\hline Total & 57 & 100 & 57 & 100 & \\
\hline
\end{tabular}

Table 3: USG, CT scan and ERCP/operative finding correlation in diagnosing cause of biliary obstruction

\section{USG diagnosis}

\section{ERCP/operative finding}

(+)ve for gallbladder mass

$\begin{array}{ll}(n=39) & \\ (n=18) & \\ & \\ & \\ (n=55) & \\ (n=2) & \text { Total }(n=57) \\ & \end{array}$

TP (39)

FN (18)

57

(-)ve for gallbladder mass

\section{Positive for diagnosis}

Negative for diagnosis

CT diagnosis

Positive for diagnosis

Negative for diagnosis

$$
\begin{aligned}
& \text { TP (55) } \\
& \text { FN (2) }
\end{aligned}
$$

FP (0)

TN (0)

0

FP (0)

TN (0) 
obstructive jaundice among the different sexes., ${ }^{9,10}$ Though in the present study, the male population was slightly more, it may be attributed to the fact that females in our country have less access to treatment facilities.

Biliary colic, jaundice, itching, abdominal lump, fever, vomiting, anorexia and weight loss are common symptoms in biliary obstruction. 9,10 $^{2}$

Serum bilirubin and alkaline phosphatase were higher in $100 \%$ of the subjects. In earlier study, all the patients had serum bilirubin level above $3 \mathrm{mg} / \mathrm{dl}$ and mean serum bilirubin level was $8 \mathrm{mg} / \mathrm{dl} .{ }^{11}$ In obstructive jaundice, serum alkaline phosphatase is usually more than three times of the upper limit of normal (40-125 U/l). ${ }^{12}$ In this series, the mean serum alkaline phosphates level was well above three times that of the normal value.

In this study, the sensitivity, specificity, accuracy, positive predictive value (PPV) and negative predictive value (NPV) of USG in evaluation of choledocholithiasis were 62.5, 100, 94.7, 100 and 94.2\% respectively, whereas the sensitivity, specificity, accuracy, PPV and NPV of CT scan in diagnosing choledocholithiasis were 75, 100, 96.5, 100 and $96.1 \%$ respectively. USG missed some cases of CBD calculi, as there was inadequate visualization of the entire CBD due to bowel gas and obesity. In a study, USG correctly defined the cause of biliary obstruction in $71 \%$ of the patients with ductal stone. ${ }^{13}$

Another study showed the sensitivity and accuracy of USG to detect choledocholithiasis 18 and 19\% respectively, whereas sensitivity and accuracy of CT ware 87 and 84\% respectively. ${ }^{14}$ Sonography was limited in its ability to image calculi in the distal CBD. Previous study ${ }^{15}$ indicated that sensitivity of sonogrpahy in the detection of CBD stones was rather poor, with only $22 \%$ cases interpreted as positive. Perhaps a calculus requires a significant amount of surrounding bile for sonographic contrast in order to be visualized as separate from the duct wall and periductal soft tissue. ${ }^{15}$ Reflection and refraction of the sound beam by curved walls of the duct also may contribute to the poor sensitivity. The common duct may be outside the optimal focal zone of the higher frequency transducers needed to image small calculi.

In this study, sensitivity, specificity, accuracy, PPV and NPV of USG were 80.0, 97.6, 93, 92.3 and 93.2\% respectively in evaluation of Ca pancreas, which were 93.3, 97.6, 96.5, 93.3 and 97.6\% respectively in CT scan. In earlier study, USG was $97 \%$ sensitive with $100 \%$ PPV, ${ }^{16}$ accuracy of USG was $80.0 \%$ and that of CT scan was $93 \%{ }^{17}$ in diagnosing pancreatic carcinoma. Their results are near similar to the present study.

More than one-third (35.1\%) patients of the present study group were ultimately diagnosed with a gallbladder mass.
USG detected 21 (36.6\%) and CT 20 (35.1\%) patients having a gallbladder mass. The sensitivity in the present study as regarding the validity of USG as a diagnostic modality in the evaluation of gallbladder mass was 95\%. This value is near to the finding of Khalili and Wilson $(2005)^{18}$ where sensitivity of USG was $94 \%$. Yeh $(1979)^{19}$ observed the accuracy of USG in the diagnosis of gallbladder carcinomas to be $84.6 \%$. The present study showed higher accuracy which was $93 \%$. In the present study specificity, PPV and NPV of USG in the diagnosis of gallbladder mass were 94.6, 90.5 and 97.2\% respectively.

Regarding validity of CT as a diagnostic modality in the evaluation of gallbladder mass in the present study, the sensitivity, specificity, accuracy, positive and negative predictive value were all 100\%. Ghafoor (2006) ${ }^{20}$ observed 93.3\% sensitivity, Kumran et al (2002) ${ }^{21}$ found accuracy of CT in the diagnosis of GB mass 93.3\%, Yoshimitsu et al $(2002)^{22}$ found sensitivity $80 \%$ and accuracy $86 \%$ in detecting gallbladder mass. Probably CT scan machines used in the present study (dual slice sub-second scan) increased the detection rate of gallbladder masses.

The sensitivity, specificity, accuracy, PPV and NPV of CT scan in diagnosing periampullery carcinoma were each $100 \%$. Upadhyaya et al (2006) ${ }^{9}$ also observed that all cases of periampullary carcinoma were detected by CT scan in their study. Lindsell (1990) ${ }^{23}$ similarly observed in his study that USG could not detect any one of the three cases of periampullary carcinoma. The mass is often obscured by gas within the duodenum and could be seen on USG only if very large.

In this study, USG could detect overall causes of biliary obstruction in 68.4\% whereas CT scan detect 96.5\% ( $p<0.001$ ). The accuracy of USG was $68.4 \%$ and that of CT scan $96.5 \%$ in detecting the cause of obstructive jaundice. Earlier study showed that CT scan had 85.71\% accuracy and USG 77\% accuracy for assessing the cause of biliary obstruction. According to them MRCP had the highest accuracy (87.5\%). ${ }^{9}$

In earlier studies, the accuracy of USG and CT scan in detecting the cause of biliary obstruction was much lower. Previous study showed that accuracy of the CT scan was $70 \%$ and that of USG was only $38 \%{ }^{1}$ However in 1981, a study $^{2}$ showed 94\% accuracy of CT in detecting the cause of biliary obstruction. In 1979, a study ${ }^{23}$ showed that a full diagnosis of the cause of jaundice was achieved in 58\% of patients. In another study, ${ }^{24}$ it was found that the cause of biliary obstruction was correctly predicted in $23 \%$ of patients. A study ${ }^{25}$ found that the cause of biliary obstruction was correctly indicated by USG in $88 \%$ and by CT scan in $63 \%$ cases. These findings contradict those of other available 
studies.May be due to the advancement of technology, CT scan has gained more accuracy.

In a study in $1993,{ }^{26}$ only $55 \%$ accuracy of USG in detecting the cause of biliary obstruction was found. In the present study, the sensitivity and PPV of USG in detecting cause of biliary obstruction has been found to be 68.4 and $100 \%$ respectively and that of CT scan as 96.5 and $100 \%$ respectively. The NPV is $0 \%$ in both examinations.

From the results of the present findings as well as findings obtained by a number of investigators, it is conceivable that both USG and CT scan are ideal and accurate diagnostic imaging modalities to detect biliary tree dilatation. However, CT scan is of greater value in detecting the cause of biliary obstruction and in evaluating the extent and involvement of surrounding structures, thus enabling planning of further management of the patients.

USG and CT are invaluable for evaluating patients with obstruction of the biliary tree. CT scan is superior to USG in detecting the cause of biliary obstruction. CT scan is rapidly emerging as the preferred technique of choice for hepatobiliary imaging. An important reason is that, whereas USG is a targeted examination, CT scan offers a more comprehensive analysis of the liver and extrahepatic abdomen and pelvis, thus providing detailed information about the extent of a lesion. Another advantage of CT scanning is that it is much less dependent on the operator's skill. With technical advances, the time required for a CT examination continues to decrease, whereas it is hard to reduce the time needed for an ultrasound examination.

\section{REFERENCES}

1. Baron RL, Stanley RJ, Lee JKT, et al. A prospective comparison of the evaluation of biliary obstruction using computed tomography and ultrasonography. Radiology 1982;145;91-98.

2. Pedrosa CS, Casanova R, Rodriguez R. Computed tomography in obstructive jaundice. Radiology 1981;139:627-34.

3. Sherlock S, Dooley J (Ed). Diseases of the liver and biliary system. (10th ed), Blackwell Science, London 2002:409-12.

4. Bressler EL, Rubin JM, McCracken S. Sonographic parallel channel sign: A reappraisal. Radiology 1987;164:343-46.

5. Cooperberg PL, Li D, Wong P, Cohen MM, Burhenne HJ. Accuracy of common hepatic duct size in the evaluation of extrahepatic biliary obstruction. Radiology 1980;135:141-44.

6. Meire HB, Cosgrove DO, Dewbury KC, Farrant P (Eds). Abdominal and general ultrasound, Churchill Livingstone (2nd ed), London 2001;299-355.

7. Haaga JR, Lanzieri CF, Gilkison RC. Computed tomography and magnetic resonance imaging of the whole body (4th ed), Mosby, USA 1998:1341-481.

8. Lee JKT, Sagel SS, Stanley RJ, Heiken JP (Eds). Computed body tomography and MRI correlation (3rd ed), Lippincott, New York 1998;779-844.
9. Upadhyaya V, Upadhyaya DN, Ansari MA, Shukla VK. Comparative assessment of imaging modalities in biliary obstruction. Ind J Radiol Imag 2006;1(4)577-82.

10. Ferrari RS, Fantozzi F, Tasciotti L, Vigni F, Francisca S, Fransi P. US, MRCP, CCT and ERCP: A comparative study in 131 patients with suspected biliary obstruction. Med Sci Monit 2005;2:MT8-18.

11. Malini S, Sabel J. Ultrasonography in obstructive jaundice. Radiology 1977;123:429-33.

12. Britton J, Bickerstaff KI, Savage A. Diseases of the biliary tract. In: Morris PJ, Wood WC (Eds). Oxford Textbook of Surgery, (2nd ed), Oxford University Press 2000;1685.

13. Rigauts H, Marchal G, Vansleenbergen W, Ponette E. Comparison of ultrasound and ERCP in the detection of common cause of obstructive biliary disease. Rofo 1992; 156(3):252-57.

14. Mitchell SE, Clark RA. A comparison of computed tomography and sonography in choledocholithiasis. AJR 1984;142(4)72973.

15. Einstein DM, Lapin SA, Ralls PW, Halls JM. The Insensitivity of sonography in the detection of choledocholithiasis'. AJR Philadelphia, USA 1984;172-73.

16. Lindsell DRM. Ultrasound imaging of pancreas and biliary tract. Lancet 1990;335:390-93.

17. Thomas MJ, Pellegrini CA, Way LW. Usefulness of diagnostic tests for biliary obstruction. Am J Surg 1982;144(1):102-08.

18. Khalili K, Wilson SR. The Biliary tree and gallbladder. In: Rumack CM, Wilson SR, Charboneau JW (Eds). Diagnostic Ultrasound (3rd ed), Elsevier, Mosby 2005;142:725-28.

19. Yeh H. Ultrasonography and computed tomography of carcinoma of the gallbladder'. Radiology 1979;133:167-73.

20. Ghafoor N. Role of ultrasound and computed tomography in the evaluation of gallbladder malignancy. MD Thesis, BIRDEM, Dhaka, Bangladesh 2006.

21. Kumran V, Gulati S, Paul B, Pande K, Sahni P, Chattopadhyay $\mathrm{K}$. The role of dual-phase helical CT in assessing resectability of carcinoma of the gallbladder. Eur Radio 2002;12(8):1993-99.

22. Yoshimitsu K, Handa H, Shinozaki K, Aibe H, Kuroiwa T, Irie $\mathrm{H}$, et al. Helical CT of the local spread of carcinoma of the gallbladder. Amer J Roengen 2002;179:423-28.

23. Dewbury KC, Joseph AEA, Hayes S, Murray C. Ultrasound in the evaluation and diagnosis of jaundice. British J Radiol 1979;52:276-80.

24. Honickman SP, Mueller PR, Wittenberg J, Simeone JF, Ferrucci JT, Cronan JJ, Sonnenberg EV. Ultrasound in obstructive jaundice: Prospective evaluation of site and cause. Radiology 1983;147:511-15.

25. Gibson RN, Yeung E, Thompson JN, Carr DH, Hemingway AP, Bradpiece HA, et al. Bile duct obstruction: Radiologic evaluation of level, cause and tumor resectability. Radiology 1986;160:43-47.

26. Dixit VK, Jain AK, Agrawal AK, Gupta JP. Obstructive Jaundice-a diagnostic appraisal. J Assoc Physicians India 1993;41(4):200-02.

\section{ABOUT THE AUTHORS}

\section{Naffisa Adedin (Corresponding Author)}

Registrar, Department of Radiology and Imaging, BIRDEM Shahbagh, Bangladesh, e-mail: naffisa.abedin@yahoo.com 


\section{Abdullah Shahriar}

Assistant Professor, Department of Pediatric Cardiology, NICVD Shere Banglanagar, Dhaka, Bangladesh

\section{Akhtar Uddin Ahmed}

Professor, Department of Radiology and Imaging, BIRDEM Shahbagh, Bangladesh

\section{AS Mohiuddin}

Professor, Department of Radiology and Imaging, BIRDEM Shahbagh, Bangladesh

\section{Jafreen Sultana}

Associate Professor, Department of Radiology and Imaging, BIRDEM Shahbagh, Bangladesh

\section{Nusrat Ghafoor}

Specialist, Department of Radiology and Imaging, Ibrahim Cardiac Hospital, Dhaka, Bangladesh

\section{Nayeema Rahman}

Junior Consultant, Department of Radiology and Imaging, BIRDEM Shahbagh, Bangladesh 